\title{
Big Sensory Data Transmission Architecture for Cold Chain Logistics
}

\author{
Chao Meng ${ }^{1}$, Long $\mathrm{Jin}^{2}$ and Zhixin Sun ${ }^{1, *}$ \\ ${ }^{1}$ School of Modern Posts\&Institute of Modern Posts, Nangjing University of Posts and Telecommunications, Nangjing210023, \\ China \\ ${ }^{2}$ National Engineering Laboratory for Logistics Information Technology, YTO Express, Shanghai201705, China \\ ${ }^{*}$ Corresponding author
}

\begin{abstract}
Cold chain logistics demands a high temperature standard during transportation. In order to ensure temperature requirement, a wireless sensor network used for cool box temperature monitoring and GPS location in cold chain logistics is presented in this paper. Wireless sensor module collects sensory data, then it is uploaded to server so as to provide real time monitoring. Because the scale of the sensory data in cold chain logistics has already exceeds several petabytes with the growth in business, this paper also presents a theoretical framework which is developed to model the link correlations in cold chain logistics sensory data. The proposed architecture can effectively reduce the number of data transmission and reduce the consumption of energy. This technology is of great practical significance for sensor nodes powered by batteries. Finally, the extensive real experiment results indicate that the proposed mechanisms obtain high performance in terms of energy consumption and accuracy.
\end{abstract}

Keywords-wireless sensor networks; big data; cold; chai; transmission algorithms; correlation

\section{INTRODUCTION}

Cold Chain Logistics refers to that frozen foods have been in low temperature environment during the each link of the production, storage, transportation and sales, to ensure the food quality and reduce loss of food. A complete cold chain logistics should deal with the goods in the whole temperature monitoring in order to ensure the safety of the food. Currently, the design and implementation of sensor node and network in collecting temperature and GPS big data is popular. Cold Chain Logistics big data needs wireless sensor network to transmit it. Big data transmission puts great pressure on Wireless Sensor Networks and causes network congestion.

Recent work has shown that wireless links are not independent in WSN[1][2], and that transmissions from a transmitter to multiple-receivers are correlated. The transmission of Logistics big data faces the same problem. The finding of link correlation brings a good model for logistics WSN design, opening up new opportunities for network optimization

At present, there has been some research effort to study the correlation Framework of Data transmission[3][4] in the field of Cold chain logistics. These work are mainly concentrated on the refrigeration freezing technology, cold chain temperature monitoring system, the food tracking and back system in logistics and so on. For example, in [5], a wireless sensor used for cool temperature monitoring in logistics is presented. The proposed scheme is implemented by integrating a wireless microcontroller based on IEEE 802.15.4 standard. On the other hand, there exist some work which attempt to exploit link correlation in transmission of Logistics big data[6][7]. However, these transmission schemes of logistics data aim to find the optimum rate so as to compress redundant information in the sensor network. These studies also do not consider exploit link correlation in big data transmission of Cold Chain Logistics.

In this paper, the authors present a big data transmission scheme. This scheme achieves a tracking and tracing system. The system includes several sensor nodes designed for temperature and humidity data collection and a sink node used for data upload and ZigBee network organization in order to facilitate the monitoring of temperature and humidity of cold chain logistics. Because of lots of data to be collected and transmitted, the authors propose a correlations metric called $\rho$ that captures this degree of correlation between the data transmission links in logistics vehicle. The authors use link correlation to reduce the scale of data transmission and energy consumption of wireless communication.

$\rho$ is a 3-tuple metric that measures packet reception correlation on two links. When $\rho$ is 1 ,it means that the two links are highly correlated, zero means they are independent, and -1 means that the losses on one are highly correlated with successes on the other[8].

When the two links are positively correlated, a node is randomly selected to transmit the sampling data. When the two links are negatively correlated, we also randomly select a node to transmit it and apply correlation analysis algorithm to calculating the sampling value of another node[8]. When the two links are independent, the two nodes transmit the sampling data, simultaneously. The framework effectively reduces the number of data transmission and energy consumption of perceptive node.

\section{System Model of COld Chain Logistics}

This paper adopts the temperature monitoring system framework of cold-chain logistics which is composed of WSN, GPRS and monitoring center, as shown in Figure 1. 


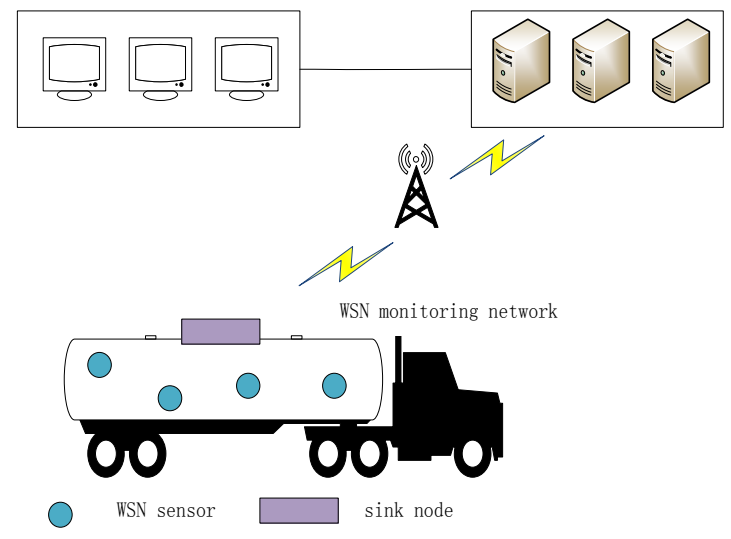

FIGURE I. COLD-CHAIN LOGISTICS NETWORK MODEL

In the cold chain Logistics transport vehicle, the temperature data is acquired by wireless sensor named as a general aware nodes, which will transmit their temperature data to the sink node deployed at the top of their vehicle. Sink node is responsible for data collection and query tasks, and process the data received. Through GPRS wireless communication, it transmits data query results to the supervision center.

The romote monitoring center has the network topology information of sink node and all aware nodes. The sink node has network topology information of all aware nodes in the vehicle. A aware node has the topology information of the sink node and neighbor aware nodes in the same vehicle.

\section{MATHEMATICAL MODEL}

The amount of data collected by perceptual nodes is huge in logistics vehicle A large number of data are needed to be stored and transferred through the network to the server side. However, the bandwidth of the network is very limited. To solve these problems, a big data transmission correlation model is presented to reduce the average transmission counts of logistics data and energy consumption of nodes.

This paper considers a popular quantity in statistics that measures correlation between two data transmission links: the cross-correlation index $\rho$.

$\rho$ is a 3-tuple of one receiver, $r$ and two random variables, $\mathrm{x}$ and $\mathrm{y}$, corresponding to transmission at two transmitters. The receiver, $r$, is sink node. " $x$ " and " $y$ " represent the two sampling node and transmit big data to sink. It assumes that " $x$ " and " $y$ " are random variables representing 1 for a successful reception and 0 for a failure. This paper uses " $x$ " and " $y$ " to indicate the transmitter and their corresponding transmission link.

This paper analysis with a simple network of 3 nodes, namely $\mathrm{x}, \mathrm{y}$ and $\mathrm{r}$. In this setup, $\mathrm{x}$ and $\mathrm{y}$ is the source node and $\mathrm{r}$ is the destination node(sink node). Figure 2 shows this setup along with the packet reception ratios of all the links.

"x" and " $y$ " are the random variables.

$$
x \sim B\left(1, P_{x}\right), y \sim B\left(1, P_{y}\right)
$$

where $P_{x}$ represents packet reception ratio(PRR), which is the probability of the successful acceptance of a data packet. Similarly, $P_{y}$ is the same meaning.

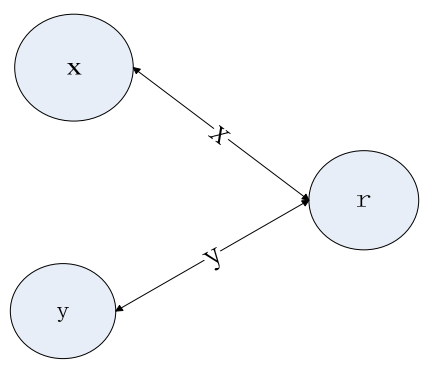

FIGURE II. A 3-NODE NETWORK

$\rho$ is defined as:

$$
\rho_{x, y, r}=\frac{E\left[\left(x-\mu_{x}\right)\left(y-\mu_{y}\right)\right]}{\sigma_{x} \sigma_{y}}=\frac{E(x y)-E(x) E(y)}{\sigma_{x} \sigma_{y}}
$$

where $\sigma_{x}=\sqrt{E\left[(x-E[x])^{2}\right]}$ is the standard deviation of $\mathrm{x}, E(x y)$ indicates the mean value of $\mathrm{x}$ multiplied by $\mathrm{y}$. $E(x)$ and $\mu_{x}$ are the means of $\mathrm{x}$, and $E(y)$ and $\mu_{y}$ are the means of $y$. The normalization factor is the maximum difference possible between $E(x y)$ and $E(x) E(y)$. It comes from the Cauchy-Schwarz inequality:

$$
\begin{aligned}
& E\left[\left(x-\mu_{x}\right)\right] \times E\left[\left(y-\mu_{y}\right)\right] \\
& \leq \sqrt{E\left[(x-E[x])^{2}\right] \times E\left[(y-E[y])^{2}\right]} \\
& \Rightarrow E(x y)-E(x) E(y) \leq \sigma_{x} \sigma_{y}
\end{aligned}
$$

$E(x y)$ is the probability that both $\mathrm{x}$ and $\mathrm{y}$ transmit the packet, successfully. $E(x y)$ is represented by $P_{x, y, r}(1,1)$.

According to (1), $E(x)$ and $E(y)$ are the PRRs of the links $x \longrightarrow r$ and $y \longrightarrow r$, respectively. Moreover, $\sigma_{x}=\sqrt{P_{x}\left(1-P_{x}\right)}$ and $\sigma_{y}=\sqrt{P_{y}\left(1-P_{y}\right)}$

Therefore, $\rho$ can be rewritten as:

$$
\rho_{x, y, r}=\frac{P_{x, y, r}(1,1)-P_{x} P_{y}}{\sqrt{P_{x}\left(1-P_{x}\right) P_{y}\left(1-P_{y}\right)}}, \sigma_{x} \sigma_{y} \neq 0
$$

According to the principle of Statistics, $-1 \leq \rho_{x, y, r} \leq 1$. $\rho_{x, y, r}$ also compares the probability that both links actually receive a given packet to the probability that both would receive a given packet if their receptions were independent. If the difference between these two values is zero then the receptions 
at $\mathrm{x}$ and $\mathrm{y}$ are independent, positive means positively correlated and negative means negatively correlated.

This paper uses $\rho_{x, y, r}$ to build the correlation between the two links( $\mathrm{x}$ and $\mathrm{y}$ ). When the two links are positively correlated, we randomly select a node to transmit the sampling data. When the two links are negatively correlated, we also randomly select a node to transmit it and apply correlation analysis algorithm to calculating the sampling value of another node. When the two links are independent, the two nodes transmit the sampling data, simultaneously. This transmission mechanism proposed in this paper can effectively reduce redundance data transmission and energy consumption and avoid the network congestion. This is of great practical significance for sensor nodes powered by batteries.

\section{PERFORMANCE ANALYSIS AND SimULATION EXPERIMENTS}

In order to observevarious degrees of correlation present between two links in the vehicle, this paper uses measurements and experiments from both 802.15.4 and 802.11b networks. 802.15.4 is an IEEE PHY-MAC low power, low data rate network standard with a 16 channel spectrum that overlaps the spectrum of $802.11 \mathrm{~b}$.We run 802.15.4 experiments using TinyOS running on the IntelMirage testbed, which consists of 10 Micaz nodes placed into logistics vehicle[9] [10].

Figures 3(a) and 3(b) show perfectly positively correlation between two links. $\mathrm{a}(1)$ and $\mathrm{a}(2)$ indicate the two links have same packet reception ratios(PRRs). $b(1)$ and $b(2)$ indicate they have different PRRs.

Figures 4(a) and 4(b) show perfectly negatively correlation between two links. There are two different cases: when the sum of the PRRs is 1 and the sum of the PRRs is not 1 .

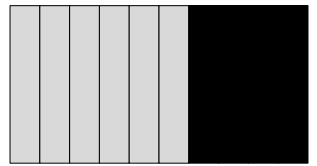

$\mathrm{a}(1)$

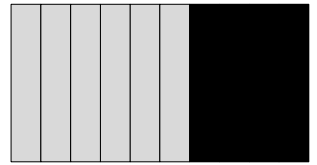

$\mathrm{a}(2)$

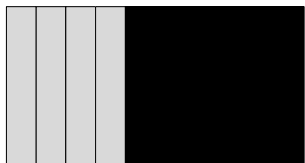

$b(1)$

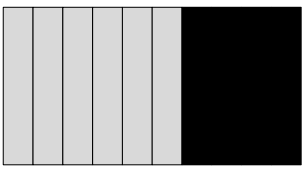

$\mathrm{b}(2)$
FIGURE III. PERFECTLY POSITIVE CORRELATION

It can be seen from the Figures 3 and Figures 4 that there exist link correlation between two links.

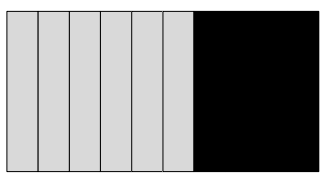

$\mathrm{a}(1)$

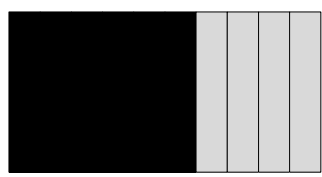

$\mathrm{a}(2)$

FIGURE IV. PERFECTLY NEGATIVE CORRELATION

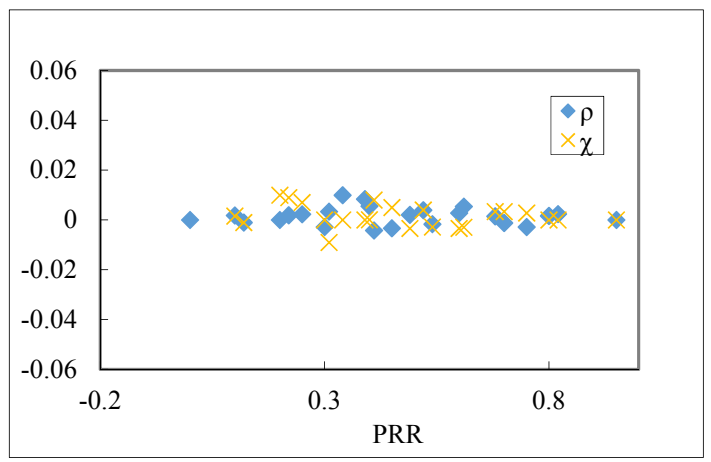

FIGURE V. INDEPENDENT
Previous paper has used cross-conditional probability, $\chi$, as the correlation [11]. This paper use a simple example to indicate why $\chi$ is not a good correlation metric.

We generate a synthetic trace on two links with varying PRRs. There are three cases. In the first case, the two links pair are independent. In the second case, the two links are positive correlation with the two links having same PRRs. This is the case shown in Figure 3(a).In the third case, the two links are negative correlation with the PRRs summing to 1 . This is the case shown in Figure 4(a).

Figure 5 shows calculated values for two independent links. The metric properly reflects the independence of the uncorrelated links with near zero values. However, in Figure 6, for the simplest case of positively correlated links, $\chi$ fails to identify the correlation, but rather causes confusion as to whether the links may be correlated or independent. $\rho_{x, y, r}$ proposed in this paper can better reflect the positive correlation of two links. Because of a certain error in the transmission process of the sampling value, there is the fluctuation of the value of $\rho_{x, y, r}$ in the vicinity of 1 . In general, $\rho_{x, y, r}$ shows the positive correlation of links, perfectly. Also, figure 7 shows the same problem when the two links are perfectly negatively correlation. From the experiments, it indicates transmission framework has a high feasibility and practical value. 

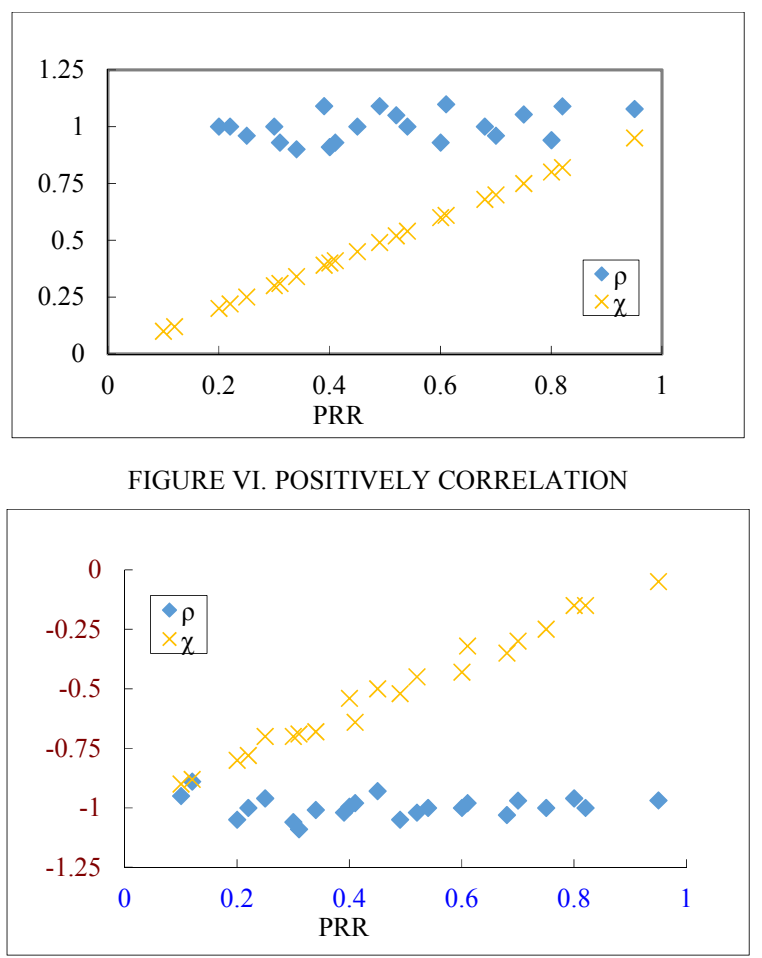

FIGURE VII. NEGATIVELY CORRELATION

The architecture proposed in this paper can effectively reduce the number of data transmission and energy consumption. The authors use expected transmission counts(ETC) to represent the scale of data transmission.

Figure 8 compares the changes of ETC in two cases of positive correlation and unrelated conditions. It can be seen from the figure that with the continuous improvement of PRR, the ETC continues to decline. This is due to the improvement of link transmission quality, which leads to the reduced number of data transmission required. In addition, under the positive correlation conditions, the number of data transmission can be greatly reduced by using the framework mechanism of this paper in contrast with uncorrelated conditions. Figure 9 also has the same problem.

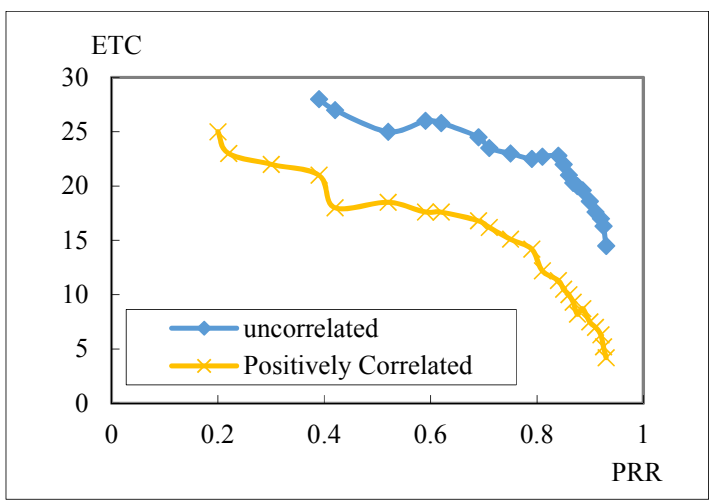

FIGURE VIII. COMPARISON OF DATA TRANSMISSION

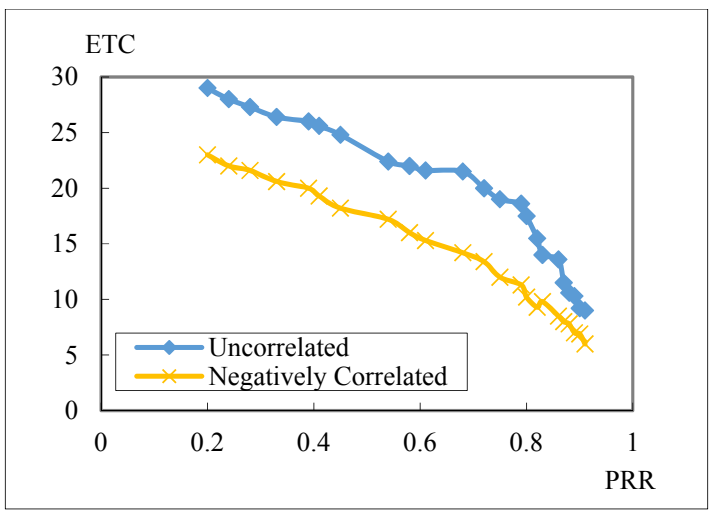

FIGURE IX. COMPARISON OF DATA TRANSMISSION

\section{SUMMARY}

The proposed framework used for data transmissionin in cold chain logistics has been developed. The link quality has also been measured in the logistics vehicle. The measured result shows that the propose framework located at the testing points in the vehicle can communicate with the sink node efficiently. The system has stable performance.

\section{ACKNOWLEDGEMENT}

This research was financially supported by the National Science Foundation(NO:61373135 61672299). Jiangsu University Natural Science Project(NO: 16KJB520033). The start-up fund project of Nanjing University of Posts and Telecommunications(NO: NY214024)

\section{REFERENCES}

[1] K. Srinivasan et al., "The -factor: Inferring protocol performance using inter-link reception correlation," in Proc. MobiCom, 2010, pp.317-328

[2] T. Zhu, Z. Zhong, T. He, and Z.-L. Zhang, "Exploring link correlation for efficient flooding in wireless sensor networks," in Proc. NSDI,2010, pp. 4

[3] TianbaoXie, Meng Zhao. Research on cold chain logistics joint distribution model based on cloudlogistics.IEEE Advanced Information Management, Communicates, Electronic and Automation Control Conference (IMCEC), 2016,pp:802-806

[4] Chen-Ming Li, Chin-Chung Nien, Jia-Liang Liao, Yu-Chee Tseng, Development of wireless sensor module and network for temperature monitoring in cold chain logistics. IEEE International Conference on Wireless Information Technology and Systems (ICWITS),2012,pp: 1-4,

[5] DasikKo, YunsikKwak, DojinChoi, Seokil Song. Design of Cold Chain Application Framework (CCAF) Based on IOT and Cloud. International Conference on $u-$ and e-Service, Science and Technology (UNESST),2015,pp: 11-13

[6] S.S. Pradhan, K. Ramchandran, Distributed source coding: symmetric rates and applications to sensor networks,in: Proceedings of the Data Compression Conference 2000,pp. 363-372.

[7] S.S. Pradhan, J. Kusuma, K. Ramchandran, Distributed compression in a dense microsensor network, IEEE Signal Process. Mag. 19 (2) (2002) 5160.

[8] Chen Wei, Xu Ruo-mei, Li Yu-ling. A Privacy-Preserving IntegrityVerification-Based Top-k Query Processing[J]. Journal of ComputerResearch and Development,2014,51(12),pp.2585-2592

[9] The Institute of Electrical and Electronics Engineers, Inc.Part 15.4: Wireless Medium Access Control (MAC) andPhysical Layer (PHY) Specifications for Low-Rate WirelessPersonal Area Networks (LRWPANs), Oct. 2003 
[10] Intel Research Berkeley. Mirage testbed. https://mirage.berkeley.intelresearch.net/

[11] A. Miu, G. Tan, H. Balakrishnan, and J. Apostolopoulos.Divert: Finegrained path selection for wireless lans. InProceedings of the Second International Conference onMobile Systems, Applications, and Services (MobiSys 2004),2004 